\title{
Multifunctional Laryngeal Premotor Neurons: Their Activities during Breathing, Coughing, Sneezing, and Swallowing
}

\author{
Keisuke Shiba, ${ }^{1,3}$ Ken Nakazawa, ${ }^{2}$ Kenichi Ono, ${ }^{1,2}$ and Toshiro Umezaki ${ }^{4}$ \\ Departments of ${ }^{1}$ Otolaryngology and ${ }^{2}$ Integrative Neurophysiology, Graduate School of Medicine, Chiba University, Chiba City, Chiba 260-8670, Japan, \\ ${ }^{3}$ Department of Otolaryngology, Chiba Medical Center, Chiba City, Chiba 260-8606, Japan, and 4Department of Otolaryngology, Graduate School of \\ Medicine, Kyushu University, Fukuoka 812-8582, Japan
}

\begin{abstract}
To examine whether motor commands of two or more distinct laryngeal motor patterns converge onto a common premotor network, we conducted dual recordings from the laryngeal adductor motoneuron and its premotor neuron within the brainstem respiratory circuitry during fictive breathing, coughing, sneezing, and swallowing in decerebrate paralyzed cats. Expiratory neurons with an augmenting firing pattern (EAUG), whose action potentials evoked monosynaptic IPSPs in the adductor motoneurons, sharply fired during the expulsive phases of fictive coughing and sneezing, during which the adductor motoneurons transiently repolarized. In contrast, these premotor neurons were silent during the swallow-related hyperpolarization in adductor motoneurons. These results show that one class of medullary respiratory neuron, EAUG, is multifunctional and shared among the central pattern generators (CPGs) for breathing, coughing, and sneezing. In addition, although the CPGs underlying these three behaviors and the swallowing CPG do overlap, EAUG neurons are not part of the swallowing CPG and, in contrast to the other three behaviors, are not a source of inhibitory input to adductor motoneurons during swallowing.
\end{abstract}

Key words: coughing; sneezing; swallowing; expiratory neuron; laryngeal motoneuron; central pattern generator

\section{Introduction}

The larynx serves various behaviors including breathing and nonrespiratory behaviors such as vocalization, straining, and airway protection reflexes (e.g., coughing, sneezing, and swallowing) (Shiba et al., 1999). How do animals use the same muscles to generate such various different behaviors? During normal breathing, laryngeal motoneurons are driven by outputs from the brainstem respiratory circuitry, which generates the breathing rhythm and breathing activity of respiratory muscles (Ezure, 1990; Ono et al., 2006). Most of the central pattern generators (CPGs) are not dedicated to producing a fixed motor pattern, but can assume different functional configurations and produce various motor patterns according to afferent inputs (Morton and Chiel, 1994; Dickinson, 1995; Marder and Calabrese, 1996). It is thus suggested that the respiratory neuronal network can be reconfigured to generate nonrespiratory behaviors of respiratory muscles including laryngeal muscles (Grélot and Bianchi, 1996; Gestreau et al., 2005).

The laryngeal movements during normal breathing are completely synchronized with respiratory rhythm; the glottis dilates

Received Aug. 18, 2006; revised March 31, 2007; accepted April 2, 2007.

This work was supported by Grants-in-Aid for Scientific Research (B) 16390485 and (C) 18591859 from the Japan Society for the Promotion of Science. We thank Dr. Alan D. Miller for comments on this manuscript.

Correspondence should be addressed to Dr. Ken Nakazawa, Department of Integrative Neurophysiology, Graduate School of Medicine, Chiba University, Chiba City, Chiba 260-8670, Japan. E-mail: shibak1963@yahoo.co.jp. D0I:10.1523/JNEUROSCI.0001-07.2007

Copyright $\odot 2007$ Society for Neuroscience $\quad$ 0270-6474/07/275156-07\$15.00/0 during inspiration and slightly narrows during postinspiration, which brakes expiratory airflow and so limits collapse of the lung (Bartlett, 1986). In contrast, the laryngeal movements during coughing, sneezing, and swallowing are not necessarily synchronized with respiratory movement identified by diaphragm and abdominal expiratory muscle activity. During the expiratory phases of coughing and sneezing when the abdominal muscles continue to be active, the glottis first constricts against forcible expiratory behavior to increase tracheal pressure (i.e., the compressive phase), then dilates transiently to release a burst of air that expels foreign bodies from the airways (i.e., the expulsive phase), and then constricts again (i.e., the narrowing phase) (Korpáš and Tomori, 1979). These periods of asynchrony between laryngeal and respiratory movements seem to be a key to elucidating the interactions among the CPGs of breathing and nonrespiratory behaviors. In the present study, we simultaneously recorded the intracellular activity of the laryngeal motoneuron and the extracellular activity of its premotor neuron during fictive breathing, coughing, sneezing, and swallowing to examine whether two or more distinct rhythmic motor patterns of the larynx are driven by common premotor neurons in the respiratory neuronal network. We focused on the expiratory neuron with an augmenting firing pattern (EAUG neuron) in the region spanning the Bötzinger (BÖT) and pre-Bötzinger (preBÖT) complexes as an inhibitory premotor neuron of the laryngeal adductor muscle (Jiang and Lipski, 1990; Ono et al., 2006). 


\section{Materials and Methods}

All of the procedures used in this study conform to the Physiological Society of Japan Guiding Principles for the Care and Use of Animals and were approved by the University Committee for the Use of Animals in Research.

Data were obtained from nine adult cats of either sex. The animals were initially anesthetized with halothane $(1.0-3.0 \%)$ vaporized in $50 \%$ nitrous oxide $/ 50 \%$ oxygen and were decerebrated at the precollicular level after bilateral ligation of the common carotid arteries. Dexamethasone $(1 \mathrm{mg} / \mathrm{kg}, \mathrm{i} . \mathrm{m}$.) and atropine $(0.1 \mathrm{mg} / \mathrm{kg}, \mathrm{i} . \mathrm{m}$. $)$ were administrated to minimize brain edema and to reduce secretion in the airways, respectively. The trachea was intubated, and cannulas were placed in the femoral artery to monitor blood pressure and in the femoral veins for drug administration. Mean blood pressure was maintained above $90 \mathrm{mmHg}$ when necessary using intravenous infusion of suprifen hydrochloride (Carnigen, $0.05 \mathrm{ml} / \mathrm{kg}$; Hoechst, Frankfurt, Germany). Animals were placed in a stereotaxic frame and the dorsal surface of the medulla was exposed for recording. The caudalmost $5 \mathrm{~mm}$ of cerebellum was aspirated to facilitate the insertion of electrodes. Rectal temperature was kept at $36-37.5^{\circ} \mathrm{C}$ using a heating lamp. Anesthesia was discontinued after the completion of all surgical procedures and at least $1 \mathrm{~h}$ before data collection. The animal was paralyzed with pancuronium bromide (Mioblock; initially $0.3 \mathrm{mg} / \mathrm{kg}$, i.v., then $0.15 \mathrm{mg} / \mathrm{kg} / \mathrm{hr}$ ) and artificially ventilated with room air (20-24 cycles/min). End-tidal $\mathrm{CO}_{2}$ was kept at $4-5 \%$. Bilateral pneumothoraces were made to reduce respiratory movements of the brainstem. At the end of each experiment, the animal was killed by an overdose of sodium pentobarbital.

Bipolar silver cuff electrodes were placed around the C5 phrenic, L1 abdominal, and hypoglossal (lateral branch) nerves for recording, and around both superior laryngeal nerves (SLNs) and both recurrent laryngeal nerves for stimulation. Activities of these nerves were amplified, full-wave rectified, and low-pass filtered (time constant, $1 \mathrm{~ms}$ ).

The membrane potential of the vocal cord adductor motoneuron and the extracellular potential of its premotor neuron were simultaneously recorded during fictive breathing, coughing, sneezing, and swallowing. Laryngeal motoneurons activated antidromically from the recurrent laryngeal nerve were classified as expiratory laryngeal motoneurons (ELMs) or inspiratory laryngeal motoneurons according to their expiratory or inspiratory depolarization, respectively (Barillot et al., 1990; Shiba et al., 1999). ELMs are considered to correspond to adductor motoneurons. EAUG neurons in the BÖT/preBÖT region were recorded as candidates of premotor neurons of ELMs. Synaptic connections between EAUG neurons and ELMs were examined by the spike-triggered averaging method. Recording procedures were performed as shown in Figure 1. A tungsten microelectrode (catalog \#25-08-3; tip impedance, 9-12 M $\Omega$; FHC, Bowdoin, ME) for stimulation was placed within the laryngeal motoneuron pool, as determined by recording antidromic field potentials after the stimulation of the recurrent laryngeal nerve. Another tungsten electrode for recording was inserted into the BÖT/preBÖT region contralateral (or ipsilateral in a few cases) to the motoneuron recording site to identify EAUG neurons that projected to the laryngeal motoneuron pool. The projection was confirmed by antidromic activation after the stimulation of the tungsten electrode in the motoneuron pool $(<150$ $\mu \mathrm{A}$ ) and by the collision test (see Fig. $1 \mathrm{~B}$ ). Once an EAUG neuron projecting to the motoneuron pool was identified, an ELM was impaled using a glass electrode containing $3 \mathrm{M} \mathrm{KCl}$ (tip impedance, $8-25 \mathrm{M} \Omega$ ). ELMs were identified by antidromic activation from the recurrent laryngeal nerve and by expiratory-related depolarization (Barillot et al., 1990; Shiba et al., 1999). For each ELM, the membrane potential was defined as the difference between the intracellular and extracellular potentials, using a single grounded $\mathrm{Ag} / \mathrm{AgCl}$ electrode inserted into the temporalis muscle as a reference. The membrane potentials of ELMs were averaged (>200 sweeps) using extracellular spikes of the EAUG neurons as triggers using a Cambridge Electronic Design (Cambridge, UK) 1401-plus data interface and Spike 2 software in conjunction with a computer (see Fig. 1C). Membrane potentials of ELMs and extracellular potentials of EAUG neurons were sampled at $20 \mathrm{kHz}$; nerve activities were sampled at $2 \mathrm{kHz}$. Electrical lesions ( $20 \mu \mathrm{A}$ negative direct current; $20 \mathrm{~s}$ ) were made to mark recording sites of some of the trigger neurons. The portions of brainstem including the recording sites were fixed in $10 \%$ formalin, sectioned transversely at $100 \mu \mathrm{m}$, and stained with thionine.

The postsynaptic potentials that show a fast rise time are thought to be evoked by direct monosynaptic neurons (Jankowska and Roberts, 1972; Ezure and Manabe, 1989; Jiang and Lipski, 1990). A reliable criterion of a monosynaptic connection is a $10-90 \%$ rise time of a PSP of $\leq 0.5 \mathrm{~ms}$ (Kirkwood, 1979). However, many monosynaptic postsynaptic potentials have longer rise times (Jack et al., 1971; Ezure and Manabe, 1989; Jiang and Lipski, 1990; Tian et al., 1999). When the 10-90\% rise time was $>0.5 \mathrm{~ms}$ and $\leq 1.2 \mathrm{~ms}$, we used an additional criterion of the similarity of antidromic and orthodromic latencies as shown by Ezure and Manabe (1988). We defined postsynaptic potentials whose $10-90 \%$ rise times were $>1.2 \mathrm{~ms}$ as polysynaptic evoked potentials. After the identification of a monosynaptic connection between an EAUG and ELM, the membrane potential of the ELM and the extracellular potential of the EAUG neuron were simultaneously recorded during fictive breathing, coughing, sneezing, and swallowing.

Fictive behaviors were evoked and identified as reported previously (Shiba et al., 1999). Briefly, fictive coughing and swallowing were evoked by electrical stimulation of the SLN (pulse duration, $0.2 \mathrm{~ms}$; frequency, $10 \mathrm{~Hz}$; intensity, $60-150 \mu \mathrm{A}$ ). Fictive coughing was identified by bursting activity in the abdominal nerve preceded by increased and prolonged phrenic activity (Bolser, 1991; Grélot and Milano, 1991). The fictive compressive and expulsive phases of coughing were identified by the changes in membrane potential of laryngeal motoneurons in addition to the neurogram activities (Shiba et al., 1999). Fictive swallowing was identified by bursting activity in the hypoglossal nerve (Oku et al., 1994; Umezaki et al., 1998a). Fictive sneezing was evoked by mechanical stimulation of the nasal cavity with a fine polyethylene tube, and was identified by bursting activity in the abdominal nerve preceded by increased phrenic activity (Satoh et al., 1998).

\section{Results}

We examined the synaptic connections of BÖT/preBÖT EAUG neurons with ELMs using the spike-triggered averaging method in 41 pairs. In 10 of 41 pairs, spikes of EAUG neurons monosynaptically hyperpolarized ELMs. Figure $1 C$ shows an example of a hyperpolarizing potential in an ELM locked to the triggering spikes of an EAUG neuron. The sharp and fast rising phase of hyperpolarization (10-90\% rise time, $0.7 \mathrm{~ms}$ ) indicates that this hyperpolarizing potential was a monosynaptically induced IPSP (Jankowska and Roberts, 1972; Ezure and Manabe, 1989; Jiang and Lipski, 1990). The following observations also support this. The latencies of this hyperpolarization from the EAUG spike and of antidromic activation of the EAUG neuron from the laryngeal motoneuron pool were 2.1 and $1.7 \mathrm{~ms}$, respectively. Taking a synaptic delay of $\sim 0.4-0.5 \mathrm{~ms}$ into consideration, the above results indicate that the connection was monosynaptic. The latencies and $10-90 \%$ rise times of 10 IPSPs averaged $2.0 \pm 0.3 \mathrm{~ms}$ (range, 1.7-2.3 ms) and $0.8 \pm 0.2 \mathrm{~ms}$ (range, 0.5-1.1 ms), respectively.

The locations of four EAUG premotor neurons were identified histologically by electrical lesions (Fig. $2 \mathrm{~B}$ ). These neurons were found ventral or ventromedial to the retrofacial nucleus, which is defied as the Bötzinger complex, or among the rostralmost part of the nucleus ambiguus and ventrolateral reticular formation, which is defied as the pre-Bötzinger complex (Schwarzacher et al., 1995).

\section{Fictive breathing}

During fictive breathing, ELMs rapidly depolarized at the transition from the inspiratory to expiratory phase and then gradually repolarized throughout the expiratory phase as shown in Figure 1 . The membrane potential rapidly hyperpolarized at the start of the phrenic activity and remained hyperpolarized throughout the 
inspiratory phase. However, EAUG neurons corresponding to premotor neurons of ELMs fired during the second half of expiration (Figs. 1A, 3A) or throughout expiration (Fig. 2C).

\section{Fictive coughing}

We successfully conducted simultaneous recordings of the intracellular potential of the ELM and extracellular potential of its premotor neuron during fictive coughing in 7 of 10 pairs. SLN stimulation induced two types of fictive coughing. The laryngeal motor pattern of type A coughing consisted of the fictive inspiratory, compressive, expulsive, and narrowing phases represented as shown in Figure 2 $\mathrm{Ca}$ (Shiba et al., 1999). ELMs hyperpolarized during the augmented phrenic discharge (inspiratory phase) and then depolarized strongly at the transition from the inspiratory to the expiratory phase (compressive phase). The membrane potential repolarized transiently after this depolarization (expulsive phase), then depolarized again for the remainder of the abdominal burst (narrowing phase). The coughing motor pattern often lacked the expulsive phase: the transient repolarization after the transition from inspiration to expiration during fictive coughing did not appear or was very weak and obscure (type B coughing) (Fig. 2Cb). In two of the paired recordings, SLN stimulation induced both types of coughing (Fig. 2). In the other five pairs, SLN stimulation induced only type A coughing in two pairs and only type B in three pairs.

During type A coughing, all four recorded EAUG premotor neurons sharply fired during the expulsive phase when ELMs repolarized transiently (Fig. 2Ca). During type B coughing, all five recorded EAUG neurons were silent during the whole expiratory phase (Fig. $2 \mathrm{Cb}$ ) or activated during the latter half of the narrowing phase.

\section{Fictive sneezing}

We successfully conducted dual recordings from the ELM and its premotor neuron during fictive sneezing in 5 of 10 pairs. In all recorded ELMs, changes in membrane potential during fictive sneezing evoked by nasal stimulation were similar to that during type A coughing (Fig. 3). After hyperpolarization during the inspiratory phase, ELMs strongly depolarized at the transition from the inspiratory to expiratory phase (i.e., the fictive compressive phase), and then repolarized briskly during the fictive expulsive phase. When sneezing was consecutively evoked by nasal stimulation, the sneezing motor pattern lacked the narrowing phase. No ELMs exhibited continuous depolarization throughout the abdominal burst, as was seen in type B coughing.

Firing patterns of EAUG laryngeal premotor neurons during sneezing were similar to that during coughing; all recorded EAUG neurons fired sharply during the fictive expulsive phase of sneezing (Fig. 3). EAUG premotor neurons were virtually silent during the fictive compressive phase.

\section{Fictive swallowing}

We conducted dual recordings from the ELM and its premotor neuron during fictive swallowing in 8 of 10 pairs. ELMs briefly hyperpolarized at the onset of swallow-related hypoglossal burst, and then strongly depolarized at the termination of the hypoglossal burst. EAUG inhibitory premotor neurons were not activated during the swallow-related hyperpolarization and completely silent throughout fictive swallowing. Figure $2 C c$ shows that expiratory-related firings of an EAUG neuron were completely suppressed during fictive swallowing.

\section{Discussion}

The present study showed that, although behaviorally distinct, the motor patterns of the larynx used common premotor neurons. EAUG premotor neurons helped shape ELM activity not only during eupnic expiration, but also during the expulsive phases of coughing and sneezing using their inhibitory monosynaptic connections. This inhibition of adductor motoneurons during the expulsive phase plays an important role in the generation of explosive expiratory airflow during coughing and sneezing (Shiba et al., 1999). In contrast to these behaviors, EAUG neurons were silent during the swallow-related hyperpolarization of ELMs. The swallow-related inhibition preceding excitation in adductor motoneurons (Gestreau et al., 2000) is important for temporal organization of the swallowing motor sequence (Zoungrana et al., 1997). However, the laryngeal motor pattern of swallowing did not use EAUG inhibitory neurons as an inhibitory synaptic source, although the silence of EAUG neurons might indirectly contribute to the swallow-related excitation of ELMs. 
A (a) spike-triggered averaging

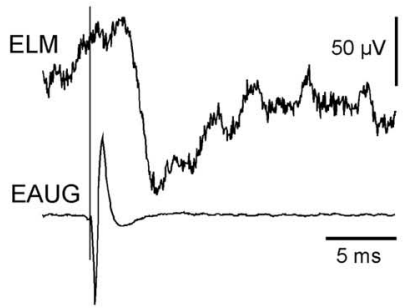

(b) collision test

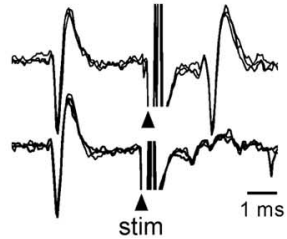

B

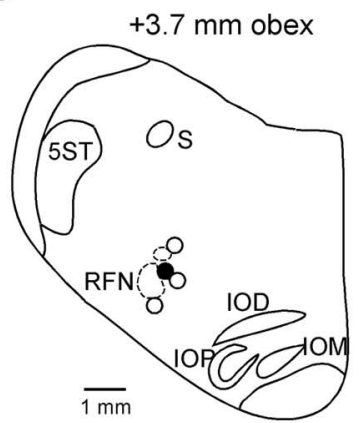

C

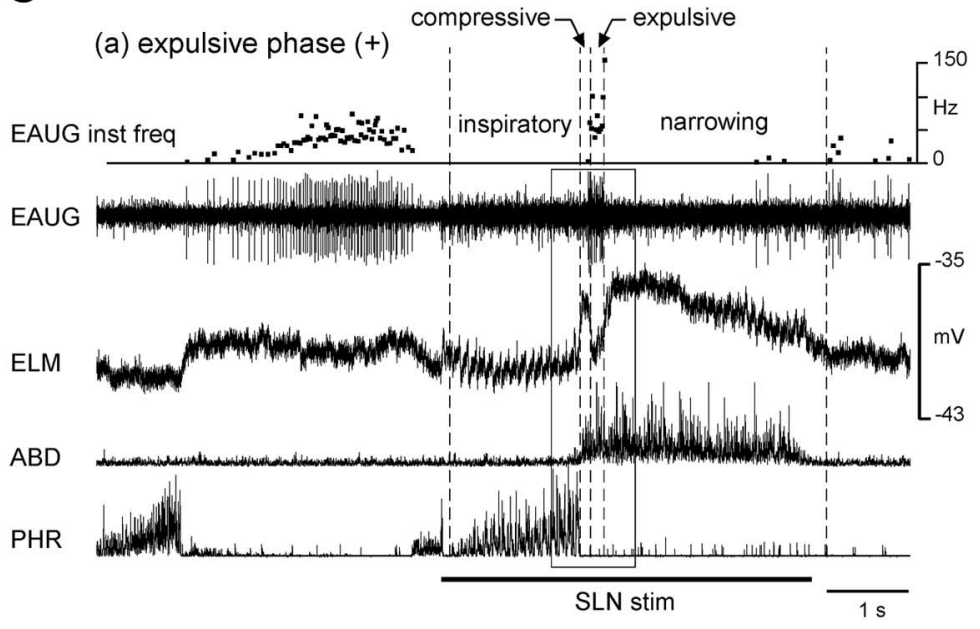

(a')

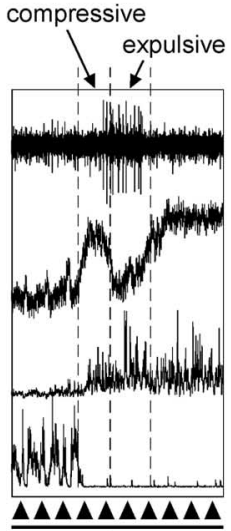

$1 \mathrm{~s}$ (c) swallowing

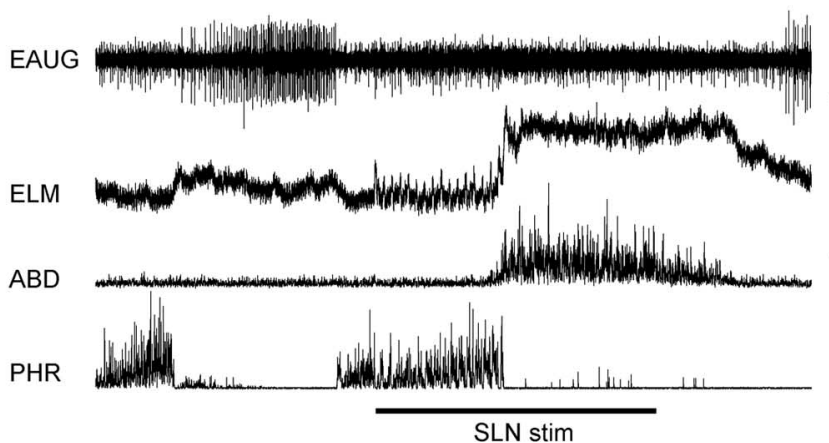

EAUG

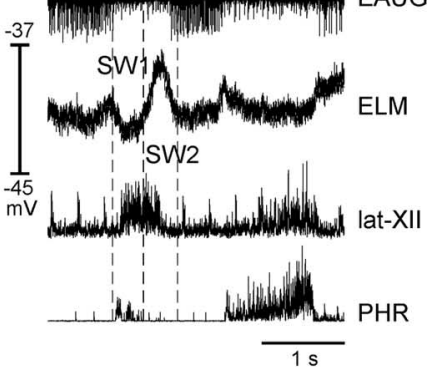

Figure 2. Activities of a laryngeal premotor neuron during fictive coughing and swallowing. $A$, An EAUG neuron-induced monosynaptic IPSP in an ELM. $\boldsymbol{a}$, Averaged membrane potential of the ELM and averaged triggering spike of the EAUG neuron ( 824 sweeps). $\boldsymbol{b}$, Axonal projection of the EAUG neuron to the region of the recorded ELM is examined by the collision test ( 3 superimposed sweeps each). Arrowheads indicate stimuli applied to the recording site of the ELM. $\boldsymbol{B}$, Locations of this EAUG neuron $(\mathbf{O})$ and three other EAUG premotor neurons $(\bigcirc)$. Neurons are projected on a transverse plane at the caudal level of the retrofacial nucleus (RFN) corresponding to the Bötzinger complex. Neurons located at the level of the rostralmost part of the nucleus ambiguus, which corresponds to the pre-Bötzinger complex, are also projected on this plane. The location of the rostralmost nucleus ambiguus is also projected on this plane as a small dotted circle above the retrofacial nucleus. IOD, Dorsal nucleus of the inferior olive; IOM, medial nucleus of the inferior olive; IOP, principal nucleus of the inferior olive; S, solitary tract; $5 \mathrm{ST}$, spinal trigeminal tract. C, Changes in extracellular potential of the EAUG premotor neuron, membrane potential of the ELM, and phrenic (PHR) and abdominal (ABD) nerve activity during fictive coughing and swallowing evoked by SLN stimulation. In this recording, SLN stimulation induced two types of coughing motor patterns: one generated the fictive expulsive phase $(\boldsymbol{a})$ and the other virtually lacked this phase $(\boldsymbol{b})$. The period indicated by the rectangular box $(\boldsymbol{a})$ is expanded on the time axis and shown in the top right corner $(\boldsymbol{a})$. When the coughing motor pattern was the former, the ELM hyperpolarized during the fictive inspiratory phase of coughing (labeled as "inspiratory"), depolarized briefly after the termination of the inspiratory phase (the fictive compressive phase labeled as "compressive"), repolarized transiently (the fictive expulsive phase labeled as "expulsive"), and then depolarized again (the fictive narrowing phase labeled as "narrowing"). Periods of SLN stimulation (duration, 0.2 ms; frequency, $10 \mathrm{~Hz}$; intensity, $100 \mu \mathrm{A}$ ) are indicated by horizontal thick lines below PHR recordings or by small arrowheads. Fictive swallowing identified by the hypoglossal burst was also evoked by SLN stimulation (c). In this figure, the neurogram of the lateral branch of the hypoglossal nerve (lat-XII) is shown instead of that of the abdominal nerve. The membrane potential briefly hyperpolarized before the swallow-related hypoglossal burst (SW1) and then abruptly depolarized for the remainder of the hypoglossal burst (SW2). This fictive swallowing was caused during the expiratory phase after cessation of SLN stimulation.

An important issue in the motor control of these respiratory and nonrespiratory behaviors is whether the same respiratory network can be reconfigured to generate various nonrespiratory behaviors, or whether each behavior requires a dedicated network. The neuronal circuit reorganization of several types of CPGs results from changes in the intrinsic membrane properties or synaptic connectivity of the circuit, and therefore causes multiple neurons in the circuit to switch from one pattern to another (Marder and Thirumalai, 2002; Nusbaum and Beenhakker, 2002). The concept of reorganizing circuitry involves overlap between multiple CPGs. The present study suggests that afferent inputs from the upper airway reconfigure the breathing CPG to evoke coughing and sneezing, because premotor neurons were shared among the CPGs of breathing, coughing, and sneezing. However, our data cannot directly address these questions. What the dual recording method can prove are direct synaptic linkages between two neurons and the assessment of whether distinct laryngeal motor patterns converge onto a common premotor network. Our experimental approach limits the ability to determine the full extent of the interactions of CPGs. To properly assess whether the respiratory network is reconfigured would require the use of imaging and electrode arrays in which multiple neurons can be simultaneously monitored. These types of studies have been conducted mainly in the invertebrate nervous system, but are beginning to be conducted also in the vertebrate medullary respiratory nervous system. Shannon et al. (2000) and Baekey et al. (2001) have conducted multiple extracellular recordings from respiratory neurons during coughing in decerebrate cats, and argued that the respiratory network is reorganized to generate the coughing motor pattern of the respiratory muscles, including the laryngeal muscles. Lieske et al. (2000) have also shown the possibility that multiple breathing patterns including 
eupnea, sigh, and gasp derived from a single respiratory network using a stereotaxic mapping technique in the brainstem slices of neonatal mice. The studies of premotor control of tongue muscle motoneuron activity also suggest that a single respiratory network can be reconfigured to produce nonrespiratory behaviors (Gestreau et al., 2005).

Oku et al. (1994) have reported that BÖT/preBÖT EAUG neurons exhibit two types of responses to SLN-induced coughing: the EAUG neurons briefly fire only after the inspiratory termination, probably during the expulsive phase, or continuously burst throughout the expiratory phase. In the present study, the bursting activity throughout cough-related expiration was never observed in the EAUG laryngeal premotor motoneurons; all recorded premotor neurons fired only during the expulsive phase or were silent during coughing. These findings indicate that BÖT/preBÖT EAUG neurons are composed of at least two neuron groups, which have different functions. Some BÖT/preBÖT EAUG neurons send their axon to the phrenic motoneurons and to various medullary inspiratory neurons, including the bulbospinal inspiratory neurons, and inhibit them monosynaptically (Merrill and Fedorko, 1984; Ezure, 1990; Jiang and Lipski, 1990; Tian et al., 1999). Phrenic motoneurons are powerfully inhibited during the expiratory phase of coughing (Grélot et al., 1992). It is thus suspected that the EAUG neurons that exhibit continuous bursting activity throughout cough-related expiration function primarily as an inhibitory source of phrenic motoneurons. However, Baekey et al. (2001) have reported the cough-related activities of three BÖT/preBÖT EAUG neurons whose spikes caused offset troughs in the rectified averages of recurrent laryngeal nerve activity; one of three neurons exhibited an increased firing rate during the expulsive phase and the other two neurons fired throughout the expiratory phase. The spike-triggered averaging of the recurrent laryngeal nerve neurogram cannot determine whether inspiratory or expiratory laryngeal motoneurons evoke the changes in activity of the recurrent laryngeal nerve, which contains both their axons, and cannot detect the IPSPs in laryngeal motoneurons when the laryngeal motoneurons do not fire. These factors may result in the discrepancy between our data and those of Baekey et al (2001).

Some previous studies have reported sneeze-related behaviors of respiratory neurons (Batsel and Lines, 1975, 1978; Jakuš et al., 1985; Orem and Brooks, 1986; Wallois et al., 1992, 1997; Pantaleo et al., 2002). However, there are few studies that have analyzed the details of sneeze-related expiratory activity of respiratory neurons by comparing the activities of the compressive and expulsive phases. Only Wallois et al. (1997) have reported that EAUG neurons are activated predominantly during the expulsive phase of sneezing, as shown in the present study. Orem and Brooks (1986) have reported that almost all BÖT/preBÖT neurons are silent during sneezing in nonanesthetized awake cats. Their data may be attributed to a weak or invisible expulsive phase of sneezing such that coughing sometimes lacked the expulsive phase, as shown in Figure $2 \mathrm{Bb}$. In contrast, Pantaleo et al. (2002) reported that a BÖT/preBÖT EAUG neuron fired throughout the expiratory phase both during coughing and during sneezing without showing changes in its activity between the expulsive and compressive phases. They suggested that BÖT/preBÖT EAUG neurons are an excitatory source of expiratory efforts of coughing and sneezing. However, almost all BÖT/preBÖT EAUG neurons have inhibitory synaptic connections with various respiratory interneurons and motoneurons (Fedorko et al., 1989; Ezure, 1990; Jiang and Lipski, 1990; Tian et al., 1999; Ono et al., 2006). We think that BÖT/preBÖT EAUG neurons function as a source of inhibitory input to laryngeal and respiratory motoneurons not only during breathing, but also during coughing and sneezing.

ELMs are inhibited at the initiation of swallowing, as shown in 
Figure $2 B c$ (Shiba et al., 1999; Gestreau et al., 2000). The present study showed that BÖT/preBÖT EAUG neurons having inhibitory monosynaptic connections with ELMs are suppressed during swallowing. Eupnic activities of laryngeal motoneurons are generated by the respiratory neuronal network in the ventrolateral medulla (Ezure, 1990; Baekey et al., 2001; Ono et al., 2006). However, the majority of respiratory neurons as well as EAUG neurons are suppressed during swallowing (Umezaki et al., 1988; Oku et al., 1994; Saito et al., 2003). These findings indicate that, although some portion of respiratory neurons might contribute to the swallow-related laryngeal movements, the role of most respiratory neurons in swallowing is minor. It is suggested that the additional swallowing-network neurons are recruited to produce the swallow-related movements of the larynx (Umezaki et al., 1998b; Jean, 2001).

Our data cannot exclude the possibility that extra premotor neurons other than the members of the respiratory network are recruited to inhibit ELMs during the expulsive phase of coughing and sneezing. For example, in the expiratory muscle activity during coughing, the activity of expiratory neurons in the caudal ventral respiratory group, which determines the eupnic activity of motoneurons of expiratory abdominal muscles, does not fully account for the behaviors of expiratory motoneurons during coughing (Oku et al., 1994). The discrepancies of the activities of the expiratory premotor neurons and their motoneurons are also observed in vomiting (Miller et al., 1987), the vestibularabdominal reflex (Shiba et al., 1996), and the SLN-intercostal reflex (Jodkowski and Berger, 1988). These findings indicate that additional neurons other than expiratory neurons in the caudal ventral respiratory group are recruited to excite the abdominal muscle motoneurons during the expiratory phase of coughing and in several reflexive behaviors. Poliaček et al. (2005) argued that neurons of the medullary lateral tegmental field, the raphe nuclei, and the pontine respiratory group are essential for the configuration of the central cough motor pattern of the larynx on the basis of the experimental results of kainic acid injection into the brainstem. Furthermore, some investigators have argued that nonrespiratory neurons in the reticular formation between the nucleus ambiguus and the nucleus of the solitary tract are also involved in the CPGs of coughing (Gestreau et al., 1997), sneezing (Wallois et al., 1997), and swallowing (Umezaki et al., 1998b, 2005; Jean, 2001). It is thus suspected that the laryngeal movements during nonrespiratory behaviors such as coughing, sneezing, and swallowing are generated not only by premotor neurons in the respiratory neuronal network, but also by nonrespiratory premotor neurons.

\section{References}

Baekey DM, Morris KF, Gestreau C, Li Z, Lindsey BG, Shannon R (2001) Medullary respiratory neurones and control of laryngeal motoneurones during fictive eupnoea and cough in the cat. J Physiol (Lond) 534:565-581.

Barillot JC, Grélot L, Reddad S, Bianchi AL (1990) Discharge patterns of laryngeal motoneurones in the cat: an intracellular study. Brain Res 509:99-106.

Bartlett DJ (1986) Upper airway motor systems. In: Handbook of physiology, Sec 3, The respiratory system, Vol 2 (Cherniack NS, Widdicombe JG, eds), pp 223-245. Bethesda, MD: The American Physiological Society.

Batsel HL, Lines AJ (1975) Neural mechanisms of sneeze. Am J Physiol 229:770-776.

Batsel HL, Lines AJ (1978) Discharge of respiratory neurons in sneezes resulting from ethmoidal nerve stimulation. Exp Neurol 58:410-424.

Bolser D (1991) Fictive cough in the cat. J Appl Physiol 71:2325-2331.

Dickinson PS (1995) Interactions among neural networks for behavior. Curr Opin Neurobiol 5:792-798.
Ezure K (1990) Synaptic connections between medullary respiratory neurons and considerations on the genesis of respiratory rhythm. Prog Neurobiol 35:429-450.

Ezure K, Manabe M (1988) Decrementing expiratory neurons of the Bötzinger complex. II. Direct inhibitory synaptic linkage with ventral respiratory group neurons. Exp Brain Res 72:159-166.

Ezure K, Manabe M (1989) Monosynaptic excitation of medullary inspiratory neurons by bulbospinal inspiratory neurons of the ventral respiratory group in the cat. Exp Brain Res 74:501-511.

Fedorko L, Duffin J, England S (1989) Inhibition of inspiratory neurons of the nucleus retroambigualis by expiratory neurons of the Bötzinger complex in the cat. Exp Neurol 106:74-77.

Gestreau C, Bianchi AL, Grélot L (1997) Differential brainstem fos-like immunoreactivity after laryngeal-induced coughing and its reduction by codeine. J Neurosci 17:9340-9352.

Gestreau C, Grélot L, Bianchi AL (2000) Activity of respiratory laryngeal motoneurons during fictive coughing and swallowing. Exp Brain Res 130:27-34

Gestreau C, Dutschmann M, Obled S, Bianchi AL (2005) Activation of XII motoneurons and premotor neurons during various oropharyngeal behaviors. Respir Physiol Neurobiol 147:159-176.

Grélot L, Bianchi AL (1996) Multifunctional medullary respiratory neurons. In: Neural control of the respiratory muscles (Miller AD, Bianchi AL, Bishop BP, eds), pp 297-304. Boca Raton, FL: CRC.

Grélot L, Milano S (1991) Diaphragmatic and abdominal muscle activity during coughing in the decerebrate cat. NeuroReport 2:165-168.

Grélot L, Milano S, Portillo F, Miller AD, Bianchi AL (1992) Membrane potential changes of phrenic motoneurons during fictive vomiting, coughing, and swallowing in the decerebrate cat. J Neurophysiol 68:2110-2119.

Jack JJ, Miller S, Porter R, Redman SJ (1971) The time course of minimal excitory post-synaptic potentials evoked in spinal motoneurones by group Ia afferent fibres. J Physiol (Lond) 215:353-380.

Jakuš J, Tomori Z, Stránsky A (1985) Activity of bulbar respiratory neurones during cough and other respiratory tract reflexes in cats. Physiol Bohemoslov 34:127-136.

Jankowska E, Roberts WJ (1972) Synaptic actions of single interneurones mediating reciprocal Ia inhibition of motoneurones. J Physiol (Lond) 222:623-642.

Jean A (2001) Brain stem control of swallowing: neuronal network and cellular mechanisms. Physiol Rev 81:929-969.

Jiang C, Lipski J (1990) Extensive monosynaptic inhibition of ventral respiratory group neurons by augmenting neurons in the Bötzinger complex in the cat. Exp Brain Res 81:631.

Jodkowski JS, Berger AJ (1988) Influences from laryngeal afferents on expiratory bulbospinal neurons and motoneurons. J Appl Physiol 64:1337-1345.

Kirkwood PA (1979) On the use and interpretation of cross-correlations measurements in the mammalian central nervous system. J Neurosci Methods 1:107-132.

Korpáš J, Tomori Z (1979) Cough and other respiratory reflexes. Basel: Karger.

Lieske SP, Thoby-Brisson M, Telgkamp P, Ramirez JM (2000) Reconfiguration of the neural network controlling multiple breathing patterns: eupnea, sighs and gasps. Nat Neurosci 3:600-607.

Marder E, Calabrese RL (1996) Principles of rhythmic motor pattern generation. Physiol Rev 76:687-717.

Marder E, Thirumalai V (2002) Cellular, synaptic and network effects of neuromodulation. Neural Netw 15:479-493.

Merrill EG, Fedorko L (1984) Monosynaptic inhibition of phrenic motoneurons: a long descending projection from Bötzinger neurons. J Neurosci 4:2350-2353.

Miller AD, Tan LK, Suzuki I (1987) Control of abdominal and expiratory intercostal muscle activity during vomiting: role of ventral respiratory group expiratory neurons. J Neurophysiol 57:1854-1866.

Morton DW, Chiel HJ (1994) Neural architectures for adaptive behavior. Trends Neurosci 17:413-420.

Nusbaum MP, Beenhakker MP (2002) A small-systems approach to motor pattern generation. Nature 417:343-350.

Oku Y, Tanaka I, Ezure K (1994) Activity of bulbar respiratory neurons during fictive coughing and swallowing in the decerebrate cat. J Physiol (Lond) 480:309-324. 
Ono K, Shiba K, Nakazawa K, Shimoyama I (2006) Synaptic origin of the respiratory-modulated activity of laryngeal motoneurons. Neuroscience 140:1079-1088.

Orem J, Brooks EG (1986) The activity of retrofacial expiratory cells during behavioral respiratory responses and active expiration. Brain Res 374:409-412.

Pantaleo T, Bongianni F, Mutolo D (2002) Central nervous mechanisms of cough. Pulm Pharmacol Ther 15:227-233.

Poliaček I, Stránsky A, Szereda-Przestaszewska M, Jakuš J, Baráni H, Tomori Z, Halašová E (2005) Cough and laryngeal muscle discharges in brainstem lesioned anaesthetized cats. Physiol Res 54:645-654.

Saito Y, Ezure K, Tanaka I, Osawa M (2003) Activity of neurons in ventrolateral respiratory groups during swallowing in decerebrate rats. Brain Dev 25:338-345.

Satoh I, Shiba K, Kobayashi N, Nakajima Y, Konno A (1998) Upper airway motor outputs during sneezing and coughing in decerebrate cats. Neurosci Res 32:131-135.

Schwarzacher SW, Smith JC, Richter DW (1995) Pre-Bötzinger complex in the cat. J Neurophysiol 73:1452-1461.

Shannon R, Baekey DM, Morris KF, Li Z, Lindsey BG (2000) Functional connectivity among ventrolateral medullary respiratory neurones and responses during fictive cough in the cat. J Physiol (Lond) 525:207-224.

Shiba K, Siniaia MS, Miller AD (1996) Role of ventral respiratory group bulbospinal expiratory neurons in vestibular-respiratory reflexes. J Neurophysiol 76:2271-2279.
Shiba K, Satoh I, Kobayashi N, Hayashi F (1999) Multifunctional laryngeal motoneurons: an intracellular study in the cat. J Neurosci 19:2717-2727.

Tian GF, Peever JH, Duffin J (1999) Bötzinger-complex, bulbospinal expiratory neurones monosynaptically inhibit ventral-group respiratory neurones in the decerebrate rat. Exp Brain Res 124:173-180.

Umezaki T, Hori T, Kiyohara T (1988) Responses of medullary respiratory neurons during swallowing in the cat. Neuroscience Res [Suppl] 7:S98.

Umezaki T, Shiba K, Zheng Y, Miller AD (1998a) Upper airway motor outputs during vomiting versus swallowing in the decerebrate cat. Brain Res 781:25-36.

Umezaki T, Matsuse T, Shin T (1998b) Medullary swallowing-related neurons in the anesthetized cat. NeuroReport 9:1793-1798.

Umezaki T, Nakazawa K, Miller AD, Nakagawa T, Komune S (2005) Central pattern generator cells for swallowing in the medulla act as those for vomiting. Soc Neurosci Abstr 31:753.10.

Wallois F, Macron V, Jounieaux V, Duron B (1992) Influence of trigeminal nasal afferents on bulbar respiratory neuronal activity. Brain Res 599:105-116.

Wallois F, Bodineau L, Macron JM, Marlot D, Duron B (1997) Role of respiratory and non-respiratory neurones in the region of the NTS in the elaboration of the sneeze reflex in cat. Brain Res 768:71-85.

Zoungrana OR, Amri M, Car A, Roman C (1997) Intracellular activity of motoneurons of the rostral nucleus ambiguus during swallowing in sheep. J Neurophysiol 77:909-922. 